\title{
On Rellich's Lemma, the Poincaré inequality, and Friedrichs extension of an operator on complex spaces
}

\author{
ChIA-ChI Tung ${ }^{1}$ \\ Pier Domenico Lamberti ${ }^{2}$ \\ 1 Department of Mathematics and \\ Statistics, Minnesota State University, \\ Mankato Mankato, MN 56001, USA \\ (emeritus). \\ imggtn140outlook.com \\ ${ }^{2}$ Dipartimento di Tecnica e Gestione dei \\ Sistemi Industriali (DTG), University of \\ Padova, Stradella S. Nicola 3-36100 \\ Vicenza, Italy. \\ lamberti@math. unipd.it
}

\begin{abstract}
This paper is mainly concerned with: (i) a generalization of the Rellich's Lemma to a Riemann subdomain of a complex space, (ii) the Poincaré inequality, and (iii) Friedrichs extension of a Schrödinger type operator. Applications to the eigenfunction expansion problem associated to the modified Laplacian are also given.

\section{RESUMEN}

Este artículo se enfoca principalmente en: (i) una generalización del Lema de Rellich a un subdominio de Riemann de un espacio complejo, (ii) la desigualdad de Poincaré, y (iii) la extensión de Friedrichs de un operador de tipo Schrödinger. Se entregan también aplicaciones al problema de expansión de funciones propias asociado al Laplaciano modificado.
\end{abstract}

Keywords and Phrases: Weighted Sobolev-Schrödinger product, Friedrichs extension, resolvent mapping.

2020 AMS Mathematics Subject Classification: 46E35, 26D10, 35J10, 32C30, 35J25.

\section{(cc) BY-NC}




\section{Introduction}

A milestone of pure and applied analysis since the last century is a selection principle of F. Rellich ([15], [4, p. 414]): Given a family of $\mathscr{C}^{1}$-functions $f$ in a bounded domain $\Omega \subset \mathbb{R}^{n}$ with smooth boundary such that both the functions $f$ and their first partial derivatives are uniformly bounded in the $L^{2}(\Omega)$-norm, then $\{f\}$ contains a Cauchy subsequence with respect to the $L^{2}(\Omega)$-norm. One consequence of this far reaching result is the Rellich's Principle: The Laplacian with zero boundary condition on a bounded domain $\Omega \subset \mathbb{R}^{N}$ has a compact resolvent. Thus the eigenfunctions of the equation

$$
-\Delta \psi=\mu \psi \quad \text { in } \Omega, \quad \psi \mid \partial \Omega=0,
$$

form a complete orthogonal basis of $L^{2}(\Omega)$. In view of some recurring interest concerning the Rellich embedding property on non-flat domains ([14], [13, 3.9.3], [11]), it seems of value to consider the question as to on what general domain the Rellich embedding and its consequences will remain valid. A main purpose of this note is to propose a setting of Riemann subdomains (in a complex space) on which an affirmative answer could be sought.

In view of the fact that on a singular space analytic tools are not yet sufficiently developed ${ }^{1}$, the basic notions of the Sobolev spaces on a Riemann subdomain (see [20]) need be properly formulated (to be recalled below). Assume throughout this paper that $(D, p)$ is a (relatively compact) Riemann subdomain in a complex space $Y$ of dimension $m$, meaning that $D$ is a relatively compact open subset of $Y$ and (as a subspace of $Y$ ) admits a holomorphic map $p$ : $D \rightarrow \mathbb{C}^{m}$ with discrete fibers. Note that the pair $(D, p)$ is a Riemann domain in the sense of $[3$, p. 19] and [8, p. 135]. If in addition $p$ is a local homeomorphism, then $(D, p)$ is said to be unramified. Every complex space of pure dimension $m$ admits locally an open neighborhood (of each given point) and a finite, open holomorphic map onto a domain in $\mathbb{C}^{m}$ ([9, pp. 107-108]), hence is locally realizable as a Riemann subdomain.

Let $\mathfrak{h}$ denote a $(2 m+1)$-tuple $\left(h_{0}, h_{1}, \cdots, h_{2 m}\right)$ of locally integrable functions $h_{j}$ on $D$. Set $V:=-h_{0}$, and denote by $\mathfrak{h}$ the $(2 m+1)$-tuple obtained by replacing the initial entry by 0 (thus $\left.\underline{\mathfrak{h}}=\left(0, h_{1}, \cdots, h_{2 m}\right)\right)$. Let $\mu$ be a non-negative constant. A $(2 m+1)$-tuple $\mathfrak{h}$ (as above) is called an allowable weight for $(D, \mu)$ if each component $h_{j}, j=1, \cdots, m$, is positive a. e. on $D$, and either $(\mu, V)=(0,0)$ or $\mathfrak{c}_{0}:=\mu-\operatorname{esssup}_{D} V>0$. (For convenience) call a $2 m$-tuple $\mathfrak{h}^{\prime}=\left(h_{1}, \cdots, h_{2 m}\right)$ with positive a. e., locally integrable components an allowable weight on $D$, and set $\mathfrak{h}_{\rightarrow}^{\prime}:=\left(0, \mathfrak{h}^{\prime}\right)$. (Unless explicitly specified) in the following let $\mathfrak{h}$ denote an allowable weight for $(D, \mu)$. Let $D^{*}$ be the largest open subset of $D$ on which the map $p=\left(p_{1}, \cdots, p_{m}\right)$ is locally biholomorphic, and set $\tilde{x}_{j}:=\operatorname{Re}\left(p_{j}\right), \tilde{y}_{j}:=\operatorname{Im}\left(p_{j}\right)$, and

\footnotetext{
${ }^{1}$ See Ruppenthal [17, p. 7]) “.. whereas geometric and algebraic methods $\cdots$ are very well developed on singular spaces, most analytic tools are still missing".
} 


$$
\partial_{j}:=\frac{\partial}{\partial \tilde{x}_{k}}, \quad j=2 k-1, \quad \partial_{j}:=\frac{\partial}{\partial \tilde{y}_{k}}, \quad j=2 k, \quad 1 \leq k \leq m,
$$

on $D^{*}$. The space $\mathscr{C}^{1}(\bar{D})$ is equipped with the (weighted) Sobolev-Schrödinger product

$$
\langle w, v\rangle_{\mu, \mathfrak{h}}:=((\mu-V) w, \bar{v})_{D}+[w, v]_{D,\left(h_{1}, \cdots, h_{2 m}\right)} \quad \forall w, v \in \mathscr{C}^{1}(\bar{D}),
$$

where $[,]_{D, \mathfrak{h}^{\prime}}$ denotes the (weighted) Dirichlet product

$$
[w, v]_{\left.D, h_{1}, \cdots, h_{2 m}\right)}:=\sum_{k=1}^{m} \int_{D^{*}}\left(h_{2 k-1} \frac{\partial w}{\partial \tilde{x}_{k}} \frac{\partial \bar{v}}{\partial \tilde{x}_{k}}+h_{2 k} \frac{\partial w}{\partial \tilde{y}_{k}} \frac{\partial \bar{v}}{\partial \tilde{y}_{k}}\right) d \tilde{v} .
$$

Here $d \tilde{v}$ denotes the pull-back of the Euclidean volume form on $\mathbb{C}^{m}$ under $p$.

Clearly for all $g \in \mathscr{C}^{1}(\bar{D})$ one has

$$
\|g\|_{\mu, \mathfrak{h}}^{2} \geq \int_{D}(\mu-V)|g|^{2} d \tilde{v} \geq \mathfrak{c}_{0}\|g\|_{L^{2}(D)}^{2} .
$$

The sesqilinear form " $\langle,\rangle_{\mu, \mathfrak{h}}$ " being elliptic, defines a scalar product on $\mathscr{C}^{1}(\bar{D})$. With respect to the induced norm \|\|$_{\mu, \mathfrak{h}}$ the completion of $\mathscr{C}^{1}(\bar{D})$ gives rise to the Sobolev space $H_{\mu, \mathfrak{h}}^{1}(D)$, with induced scalar product (denoted by the same) and induced norm \|\|$_{\mu, \mathfrak{h}}$. Similarly the completion of the space $\mathscr{C}^{\infty, c}(D)$ of test functions defines the Sobolev space $H_{\mu, \mathfrak{h}, c}^{1}(D)$ (see [20] and also [19]). Observe also that the Dirichlet product

$$
[w, v]_{D,\left(h_{1}, \cdots, h_{2 m}\right)}=\langle w, v\rangle_{1,\left(0, h_{1}, \cdots, h_{2 m}\right)}-(w, \bar{v})_{D}
$$

where $w, v \in \mathscr{C}^{1, c}(\bar{D})$ (given explicitly by the equation (1.2)) extends to a scalar product on $H_{0,\left(0, h_{1}, \cdots, h_{2 m}\right)}^{1}(D)$. The norm of $f \in H_{1,\left(0, h_{1}, \cdots, h_{2 m}\right)}^{1}(D)$ is given by

$$
\|f\|_{1,\left(0, h_{1}, \cdots, h_{2 m}\right)}=\left(\int_{D}|f|^{2} d \tilde{v}+[f, f]_{D,\left(h_{1}, \cdots, h_{2 m}\right)}\right)^{\frac{1}{2}} .
$$

As an application of the inner products (1.1)-(1.2), an explicit representation of the Friedrichs extension of the weighted Schrödinger operator ${ }^{2}$ on a Riemann subdomain, allowing possibly singular points, is derived (Theorem 3.1).

It is well-known that the employment of the Poincaré inequality plays a central role in the study of compact Sobolev embeddings. The connection between the (classical) Poincaré inequality and the Rellich embedding theorem (for Euclidean domains) was clarified by Galaz-Fontes [7]. ${ }^{3}$ Given an allowable $2 m$-weight $\mathfrak{h}^{\prime}$ on a non-flat subdomain $(D, p)$, questions remain open, however, as to

\footnotetext{
${ }^{2}$ This operator is motivated by the classical Schrödinger operator (appearing in the Schrödinger equation); the latter has been considered as "one of the most interesting objects in mathematical physics ..." (see [12, p. 3]).

3 The Poincaré inequality is sometimes referred to as the Poincaré-Friedrichs inequality. An underlying incentive for this paper is provided by a motivational remark in [7] (where by the "Friedrichs inequality" is meant the "Poincaré inequality"), "an explicit connection between the "Friedrichs inequality" and the Rellichs theorem has not been reported" (at least for a Riemann subdomain).
} 
whether (either of) the following properties holds: (1) $(D, p)$ has the Poincaré property relative to $\mathfrak{h}^{\prime}$, in the sense that there exists a constant $C_{D, \mathfrak{h}^{\prime}}$ such that the following Poincaré inequality (with variable coefficients) holds:

$$
\|f\|_{L^{2}(D)} \leq C_{D, \mathfrak{h}^{\prime}}[f, f]_{D, \mathfrak{h}^{\prime}}^{\frac{1}{2}} \quad \forall f \in H_{1,\left(0, \mathfrak{h}^{\prime}\right), c}^{1}(D) ;
$$

(2) $(D, p)$ is a Rellich domain with respect to $\mathfrak{h}^{\prime}$, namely the Rellich property, "the embedding $H_{1, \mathfrak{\mathfrak { h } ^ { \prime }}, c}^{1}(D) \hookrightarrow L^{2}(D)$ is compact”.

The Rellich embedding property will be proved in $\S 4$ (independently of the Poincaré inequality for constant allowable weight) only for the case where $(D, p)$ satisfies the following conditions: (i) $(D, p)$ is of finite volume, namely, $\int_{D^{*}} d \tilde{v}<\infty$, and (ii) $(D, p)$ is of Sobolev type, that is, there exists a constant $\alpha>2$ such that, for all $f \in H_{1,(0,1, \cdots, 1), c}^{1}(D)$,

$$
\|f\|_{L^{\alpha}(D)} \leq \text { Const. }\|f\|_{1,(0,1, \cdots, 1)} .
$$

Every Euclidean domain in $\mathbb{C}^{m}$ is of Sobolev type ([6, Theorem 6, p. 270]). Other examples are provided by finitely quasiregular Riemann subdomains (see §4).

Theorem 1.1. (Rellich embedding theorem): If $(D, p)$ is of finite volume and of Sobolev type, then $(D, p)$ is a Rellich domain with respect to any constant allowable $2 m$-weight on $D$.

Theorem 1.2. If $(D, p)$ is an unramified Riemann subdomain in a complex space and if $p$ defines an étale covering ${ }^{4}$, then $(D, p)$ has the Poincaré property relative to any allowable $2 m$-weight $\mathfrak{h}^{\prime}$ on $D$ with

$$
\mathfrak{h}_{D}:=\min \left\{\operatorname{essinf}_{D}\left(h_{j}\right) \mid 1 \leq j \leq 2 m\right\}>0 .
$$

The Rellich embedding property can be extended to sections of a vector bundle over a differentiable manifold (see [10, p. 88 and p. 93], [22, p. 111]). It would be interesting to characterize those Riemann subdomains of an $m$-dimensional complex space which can be realized as a Rellich domain with respect to some allowable $2 m$-weight. A form of the Poincaré inequality (with respect to a continuous allowable $2 m$-weight) holds as a consequence of the Rellich embedding property (see Proposition 4.3).

For a Riemann subdomain $D$ with the Poincaré property relative to an allowable $2 m$-weight $\mathfrak{h}^{\prime}$, the defining norms of the Sobolev spaces $H_{1, \underset{\mathfrak{h}^{\prime}, c}{\rightarrow}}^{1}(D)$ and $H_{0, \mathfrak{\mathfrak { h } ^ { \prime }}, c}^{1}(D)^{5}$ are equivalent. On such a domain the inhomogeneous Dirichlet problem (for the Poisson equation) admits a weak solution (Corollary 4.5). As a further application, consider the following (inhomogeneous) Dirichlet problem (to be referred to as a Poisson problem):

$$
-\sum_{j=1}^{2 m} \partial_{j}\left(h_{j} \partial_{j} \psi\right)+\alpha \psi=g \quad \text { a.e. in } D, \quad \psi \mid d D=0,
$$

\footnotetext{
${ }^{4}$ Defined in $\S 4$.

${ }^{5}$ This equivalence is sometimes called the Friederichs's Lemma. The traditional notation for the Sobolev space $H_{1,(0,1, \cdots, 1), c}^{1}(D)$ is $W_{0}^{1,2}(D)$.
} 
where $g \in L^{2}(D)^{6}$. A (weak) solution on $D$ of this problem is taken in the following sense: $\psi$ is an element in $H_{1, \mathfrak{h}, c, c}^{1}(D)$ satisfying the equation

$$
-\sum_{j=1}^{2 m} \partial_{j}\left(h_{j} \partial_{j} \psi\right)+\alpha \psi=g
$$

weakly in $D$, namely, $\psi$ satisfies the functional equation

$$
[\psi, v]_{D, \mathfrak{h}^{\prime}}+\alpha(\psi, \bar{v})_{D}=(g, \bar{v})_{D}, \quad \forall v \in \mathscr{C}^{\infty, c}(D)
$$

To solve this problem one must also determine the eigenvalues $\lambda:=-\alpha$. To this end, an alternative but more expedient formulation of the above equation is available (see Lemma 6.2). The latter requires the use of an operator, to be called a resolvent map, which can be introduced as follows: for each allowable $2 m$-weight $\mathfrak{h}^{\prime}$ on $D$ (as above with respect to $\mathfrak{h}^{\prime}$ ), there is a linear mapping $\mathscr{R}_{D, \mathfrak{h}^{\prime}}: L^{2}(D) \rightarrow L^{2}(D)$ with image in $\underset{1, \mathfrak{h}, c}{H_{\mathfrak{h}}, c}(D)$ defined by the equation

$$
\left[\mathscr{R}_{D, \mathfrak{h}^{\prime}} f, v\right]_{D, \mathfrak{h}^{\prime}}=(f, \bar{v})_{D}
$$

for all $(f, v) \in L^{2}(D) \times H_{1, \underset{h^{\prime}}{\rightarrow}, c}^{1}(D)$. On a Rellich domain this map (in the special case of $\mathscr{R}_{D,(1, \cdots, 1)}$ ) is in fact a compact mapping:

Theorem 1.3. For any relatively compact Rellich domain $D$ in $Y$, the resolvent map $\mathscr{R}_{D,\{1, \cdots, 1\}}$ : $L^{2}(D) \rightarrow L^{2}(D)$ defined by

$$
\left[\mathscr{R}_{D,\{1, \cdots, 1\}} g, v\right]_{D,\{1, \cdots, 1\}}=(g, \bar{v})_{D}, \quad \forall(g, v) \in L^{2}(D) \times H_{1,(0,1, \cdots, 1), c}^{1}(D),
$$

is a compact, self-adjoint mapping.

If $(D, p)$ is a Rellich domain of type $\mathfrak{h}^{\prime}$, then the behavior of the solutions of the boundaryeigenvalue problem

$$
-\sum_{j=1}^{2 m} \partial_{j}\left(h_{j} \partial_{j} \psi\right)+\alpha \psi=0 \quad \text { a.e. in } D, \quad \psi \mid d D=0,
$$

can be determined, and similarly for the case of the Poisson problem (1.5). For completeness this spectral analysis is carried out in $\S 6$. Given mathematical physicists' interest in complexified space-time models ${ }^{7}$, it is hoped that the results of this paper may be of use in some applications.

\footnotetext{
${ }^{6}$ Here (and in the following) $d D$ denotes the (maximal) boundary manifold of $D_{\text {reg }}$ in the manifold $Y_{\text {reg }}$ of simple points of $Y$, oriented towards the exterior of $D_{\text {reg }}([18$, p. 218]).

${ }^{7}$ See, e. g., Hansen, R. O. and Newman, E. T. A complex Minkowski approach to twistors, GRG Vol.6, No.4 (1975), 361-385.
} 


\section{Preliminaries}

In what follows every complex space is assumed to be reduced and has a countable basis of topology. For the definition and basic properties of differential forms on a complex space, see [18, §4.1]. In particular, the exterior differentiation $d$, the operators $\partial, \bar{\partial}$ and $d^{c}:=(1 / 4 \pi i)(\partial-\bar{\partial})$ are welldefined ([18, Chap. 4]). For an open subset $G$ of $Y$, denote by $\mathscr{C}_{k}^{\nu}(G)$ the set of $\mathbb{C}$-valued $k$-forms of class $\mathscr{C}^{\nu}$ on $G, \mathscr{C}_{k, c}^{\nu}(G)$ the subspace of compactly supported $k$-forms (dropping the degree if $k=0$ ), with $\nu=\beta$ to mean locally bounded, $\nu=\mathfrak{m}$ measurable, and $\nu=\lambda$ locally Lipschitzian $([18, \S 4])$. Similarly for $\mathscr{C}_{k}^{\nu}(\bar{G})$. A measurable function ${ }^{8} g$ on $Y$ is said to be locally integrable $\left(g \in L_{\text {loc }}^{1}(Y)\right)$ provided the form $g \chi$ is locally integrable on $Y_{\text {reg }}$ for every $2 m$-form $\chi \in \mathscr{C}_{2 m}^{0}(Y)$. Similarly define $L^{1}(Y)$ and $L_{\text {loc }}^{2}(Y)$ (for the latter, the above $g \chi$ is replaced by $|g|^{2} \chi$ ).

Denote by $\|z\|$ the Euclidean norm of $z=\left(z_{1}, \cdots, z_{m}\right) \in \mathbb{C}^{m}$, where $z_{j}=x_{j}+i y_{j}$. Let the space $\mathbb{C}^{m}$ be oriented so that the form $v^{m}:=\left(d d^{c}\|z\|^{2}\right)^{m}$ is positive. Let $\mathbb{B}(r)$ denote the $r$-ball in $\mathbb{C}^{m}$ centered at the origin and $\mathbb{B}[1]=\left\{z \in \mathbb{C}^{m} \mid\|z\| \leq 1\right\}$. Let $p: Y \rightarrow \mathbb{C}^{m}$ be a holomorphic map. If $S \subseteq Y$, let $S^{\prime}:=p(S)$; and in particular write $a^{\prime}=p(a)$. Set $p^{[a]}:=p-a^{\prime}, \forall a \in Y$. Clearly the form

$$
v_{p}:=d d^{c}\left\|p^{[a]}\right\|^{2}=\left(\frac{i}{2 \pi}\right) \partial \bar{\partial}\left\|p^{[a]}\right\|^{2}
$$

is non-negative and independent of $a$. Denote by $d v$ the Euclidean volume element of $\mathbb{C}^{m}$ and define $d \tilde{v}:=p^{*}(d v)$ on $Y$. If $(D, p)$ is a Riemann subdomain, then $d \tilde{v}$ is a semivolume form on $D$ and

$$
d \tilde{v}=\frac{c_{m} \pi^{m}}{m !} v_{p}^{m}
$$

where $c_{m}:=(-1)^{\frac{m(m-1)}{2}}$. If $f, \psi \in L_{\text {loc }}^{1}(D)$, set

$$
(f, \psi)_{D}:=\int_{D} f \psi d \tilde{v}
$$

provided the integral exists. Each element $f \in L_{\text {loc }}^{1}(D)$ gives rise naturally to a top-dimensional current, $T=[f]: \chi \mapsto \int f \wedge \chi$, with induced functional (cf. [21]) defined by

$$
\langle[f], \phi\rangle:=(-1)^{\frac{m(m-1)}{2}} \frac{\pi^{m}}{m !} \int f \phi v_{p}^{m}, \quad \forall \phi \in \mathscr{C}^{\infty, c}(D) .
$$

\section{Friedrichs extension of a Schrödinger type operator}

Given an allowable $(2 m+1)$-weight $\mathfrak{h}$ for $(D, \mu)$ and $g \in L^{2}(D)$, the antilinear functional $v \mapsto$ $(g, \bar{v})_{D}$ is well-defined and continuous on $H_{\mu, \mathfrak{h}, c}^{1}(D)$. By the Riesz representation theorem, there is a unique element $w \in H_{\mu, \mathfrak{h}, c}^{1}(D)$ satisfying the equation

$$
\langle w, v\rangle_{\mu, \mathfrak{h}}=(g, \bar{v})_{D}, \quad \forall v \in H_{\mu, \mathfrak{h}, c}^{1}(D),
$$

\footnotetext{
${ }^{8}$ A function $g$ on $Y$ is said to be measurable on $Y$ is so is the restriction $g \mid Y_{\text {reg. }}$.
} 
with $\|w\|_{\mu, \mathfrak{h}} \leq$ const. $\|g\|_{L^{2}(D)}$. The assignment $g \mapsto G_{\mu, \mathfrak{h}} g:=w$ defines a linear, continuous mapping $G_{\mu, \mathfrak{h}}$ of $L^{2}(D)$ onto its image $\mathfrak{R}_{\mu, \mathfrak{h}}(D):=G_{\mu, \mathfrak{h}}\left(L^{2}(D)\right)$. Clearly one has

$$
\left\langle G_{\mu, \mathfrak{h}} g, v\right\rangle_{\mu, \mathfrak{h}}=(g, \bar{v})_{D}, \quad \forall v \in H_{\mu, \mathfrak{h}, c}^{1}(D) .
$$

Thus $G_{\mu, \mathfrak{b}}$ may be regarded as a (weak) Green's map for the weighted Sobolev-Schrödinger operator. Observe that

$$
\left\langle G_{\mu, \mathfrak{h}} v, v\right\rangle_{\mu, \mathfrak{h}}=\|v\|_{L^{2}(D)}^{2}, \quad \forall v \in H_{\mu, \mathfrak{h}, c}^{1}(D)
$$

and

$$
\left\langle G_{\mu, \mathfrak{h}} \psi, v\right\rangle_{\mu, \mathfrak{h}}=\overline{\left\langle G_{\mu, \mathfrak{h}} v, \psi\right\rangle_{\mu, \mathfrak{h}}}=\left\langle\psi, G_{\mu, \mathfrak{h}} v\right\rangle_{\mu, \mathfrak{h}} \quad \forall(\psi, v) \in H_{\mu, \mathfrak{h}, c}^{1}(D) \times H_{\mu, \mathfrak{h}, c}^{1}(D) .
$$

By the equation (3.3) the Green's map $G_{\mu, \mathfrak{h}}: L^{2}(D) \rightarrow H_{\mu, \mathfrak{h}, c}^{1}(D)$ is injective, and the inverse map $\mathscr{F}_{\mu, \mathfrak{h}}:=G_{\mu, \mathfrak{h}}^{-1}: \mathfrak{R}_{\mu, \mathfrak{h}}(D) \rightarrow L^{2}(D)$ is well-defined. Also, it is positive definite since given $g \in L^{2}(D)$, the equation (3.2) implies that

$$
\begin{aligned}
\left(\mathscr{F}_{\mu, \mathfrak{h}}\left(G_{\mu, \mathfrak{h}} g\right), \overline{G_{\mu, \mathfrak{h}} g}\right)_{D} & =\left(g, \overline{G_{\mu, \mathfrak{h}} g}\right)_{D} \\
& =\left\langle G_{\mu, \mathfrak{h}} g, G_{\mu, \mathfrak{h}} g\right\rangle_{\mu, \mathfrak{h}}=\left\|G_{\mu, \mathfrak{h}} g\right\|_{\mu, \mathfrak{h}}^{2} \\
& \geq \text { const. }\left\|G_{\mu, \mathfrak{h}} g\right\|_{L^{2}(D)}^{2} .
\end{aligned}
$$

For an allowable $(2 m+1)$-weight $\mathfrak{h}$ for $(D, \mu)$ of class $\mathscr{C}^{1}$ (namely, so is each component $h_{j}$ of $\mathfrak{h}$ on $D$ ), define the Schrödinger operator $\mathcal{S}_{\mu, \mathfrak{h}}$ acting on $\mathscr{C}^{1}(D)$ (in the classical sense) by

$$
\mathcal{S}_{\mu, \mathfrak{h}} \psi:=(\mu-V) \psi-\sum_{j=1}^{2 m} \partial_{j}\left(h_{j} \partial_{j} \psi\right) \text { on } D^{*} .
$$

Assume (in the following theorem) that $\mathfrak{h}$ is an allowable $(2 m+1)$-weight for $(D, \mu)$ of class $\mathscr{C}^{1}$. Define $\mathscr{D}_{\mu, \mathfrak{h}}:=\left\{\psi \in \mathscr{C}^{2, c}(D) \mid \mathcal{S}_{\mu, \mathfrak{h}} \psi \in L^{2}(D)\right\}$, and $\mathfrak{H}\left(D, \mathcal{S}_{\mu, \mathfrak{h}}\right):=H_{\mu, \mathfrak{h}, c}^{1}(D) \cap\{w \in$ $\left.L^{2}(D) \mid \mathcal{S}_{\mu, \mathfrak{h}}[w] \in L^{2}(D)\right\}^{9}$.

Theorem 3.1. (Friedrichs extension of the operator $\mathcal{S}_{\mu, \mathfrak{h}}$ ) Let $D$ be a Riemann subdomain in $Y$. Then the weighted Sobolev-Schrödinger operator $\mathcal{S}_{\mu, \mathfrak{h}}: \mathscr{D}_{\mu, \mathfrak{h}} \rightarrow L^{2}(D)$ admits a positive, selfadjoint extension $\mathscr{F}_{\mu, \mathfrak{h}}: \mathfrak{H}\left(D, \mathcal{S}_{\mu, \mathfrak{h}}\right) \rightarrow L^{2}(D)$ with the property that for each $w \in \mathfrak{H}\left(D, \mathcal{S}_{\mu, \mathfrak{h}}\right)$,

$$
\left(\mathscr{F}_{\mu, \mathfrak{h}} w, \bar{v}\right)_{D}=\langle w, v\rangle_{\mu, \mathfrak{h}}, \quad \forall v \in H_{\mu, \mathfrak{h}, c}^{1}(D),
$$

and

$$
\left(\mathscr{F}_{\mu, \mathfrak{h}} w, \bar{v}\right)_{D}=\left(w, \mathcal{S}_{\mu, \mathfrak{h}} \bar{v}\right)_{D}, \quad \forall v \in \mathscr{C}^{\infty, c}(D)
$$

Proof. It will be shown that the inverted mapping $\mathscr{F}_{\mu, \mathfrak{h}}:=G_{\mu, \mathfrak{h}}^{-1}$ acts as an extension of the operator $\mathcal{S}_{\mu, \mathfrak{h}}$ to $\mathfrak{R}_{\mu, \mathfrak{h}}(D)=G_{\mu, \mathfrak{h}}\left(L^{2}(D)\right)$. It follows from the symmetry of $G_{\mu, \mathfrak{h}}^{-1}: L^{2}(D) \rightarrow \mathfrak{R}_{\mu, \mathfrak{h}}(D)$ that $\mathscr{F}_{\mu, \mathfrak{h}}: \mathfrak{R}_{\mu, \mathfrak{h}}(D) \rightarrow L^{2}(D)$ is self-adjoint, and satisfies the equation

$$
\left(\mathscr{F}_{\mu, \mathfrak{h}} w, \bar{v}\right)_{D}=\langle w, v\rangle_{\mu, \mathfrak{h}}, \quad \forall(w, v) \in \mathfrak{R}_{\mu, \mathfrak{h}}(D) \times H_{\mu, \mathfrak{h}, c}^{1}(D) .
$$

\footnotetext{
9 " $\mathcal{S}_{\mu, \mathfrak{h}}[w]$ "] denotes the (weak) action of $\mathcal{S}_{\mu, \mathfrak{h}}$ on the functional $[w]$.
} 
If further $v$ is an element of $\mathscr{C}^{2, c}(D)$, then

$$
\langle w, v\rangle_{\mu, \mathfrak{h}}=\lim _{j \rightarrow \infty}\left\langle w_{j}, v\right\rangle_{\mu, \mathfrak{h}}=\lim _{j \rightarrow \infty}\left(w_{j}, \mathcal{S}_{\mu, \mathfrak{h}} \bar{v}\right)_{D}
$$

This implies that

$$
\left(\mathscr{F}_{\mu, \mathfrak{h}} w, \bar{v}\right)_{D}=\left(w, \mathcal{S}_{\mu, \mathfrak{h}} \bar{v}\right)_{D} \cdot
$$

To see that the mapping $\mathscr{F}_{\mu, \mathfrak{h}}$ is indeed defined on $\mathfrak{H}\left(D, \mathcal{S}_{\mu, \mathfrak{h}}\right)$ one needs to check that

$$
\mathfrak{R}_{\mu, \mathfrak{h}}(D)=\mathfrak{H}\left(D, \mathcal{S}_{\mu, \mathfrak{h}}\right) .
$$

Let $w \in \mathfrak{R}_{\mu, \mathfrak{h}}(D)$. Clearly then $w \in H_{\mu, \mathfrak{h}, c}^{1}(D)$ and $w=G_{\mu, \mathfrak{h}} g$ for some $g \in L^{2}(D)$. Then by the equation (3.8) one has, for all $v \in \mathscr{C}^{\infty, c}(D)$,

$$
\left(w, \mathcal{S}_{\mu, \mathfrak{h}} v\right)_{D}=(\psi, v)_{D}
$$

with $\psi:=\mathscr{F}_{\mu, \mathfrak{h}} w \in L^{2}(D)$, and thus $\mathcal{S}_{\mu, \mathfrak{h}}[w]=\psi$ as desired. Hence $w \in \mathfrak{H}\left(D, \mathcal{S}_{\mu, \mathfrak{h}}\right)$. Conversely if $w \in \mathfrak{H}\left(D, \mathcal{S}_{\mu, \mathfrak{h}}\right)$, then $w \in H_{\mu, \mathfrak{h}, c}^{1}(D)$ and $\mathcal{S}_{\mu, \mathfrak{h}}[w] \in L^{2}(D)$. Thus there exists an element $g \in L^{2}(D)$ such that

$$
\left(\mathcal{S}_{\mu, \mathfrak{h}}[w], v\right)_{D}=(g, v)_{D}, \quad \forall v \in \mathscr{C}^{\infty, c}(D)
$$

For each $v \in H_{\mu, \mathfrak{h}, c}^{1}(D)$ and a sequence $\left\{v_{j}\right\}$ in $\mathscr{C}^{\infty, c}(D)$ tending to $v$,

$$
\langle w, v\rangle_{\mu, \mathfrak{h}}=\lim _{j \rightarrow \infty}\left\langle w, v_{j}\right\rangle_{\mu, \mathfrak{h}}=\lim _{j \rightarrow \infty}\left(w, \mathcal{S}_{\mu, \mathfrak{h}} \bar{v}_{j}\right)_{D}=\lim _{j \rightarrow \infty}\left(g, \bar{v}_{j}\right)_{D}=(g, \bar{v})_{D}
$$

hence $w$ satisfies the equation (3.2), thereby proving that $w \in \mathfrak{R}_{\mu, \mathfrak{h}}(D)$. Therefore the formula (3.5) follows from the equation (3.7). Consequently the mapping $\mathscr{F}_{\mu, \mathfrak{h}}$ gives the Friedrichs extension of $\mathcal{S}_{\mu, \mathfrak{h}}: \mathscr{D}_{\mu, \mathfrak{h}} \rightarrow L^{2}(D)$.

Remark 3.2. Especially the preceding theorem ensures that the existence of the Friedrichs extension of the Laplacian ${ }^{10}:-\triangle_{\{p\}}: \mathscr{D}_{\mu,(1, \cdots, 1)} \rightarrow L^{2}(D)$. This extension is given by the mapping $\mathscr{F}: \mathfrak{H}\left(D,-\triangle_{\{p\}}\right) \rightarrow L^{2}(D)$, and admits the representation

$$
(\mathscr{F} w, \bar{v})_{D}=[w, v]_{D,(1, \cdots, 1)}, \quad \forall(w, v) \in \mathfrak{H}\left(D,-\triangle_{\{p\}}\right) \times H_{1,(0,1, \cdots, 1), c}^{1}(D)
$$

In particular, for all $(w, v) \in \mathfrak{H}\left(D,-\triangle_{\{p\}}\right) \times \mathscr{C}^{\infty, c}(D)$,

$$
(\mathscr{F} w, \bar{v})_{D}=\left(w,-\triangle_{\{p\}} \bar{v}\right)_{D}
$$

\section{The Rellich Embedding Theorem and Poincaré inequality}

Let $p: Y \rightarrow Y^{\prime}$ be a continuous mapping (between topological spaces). An open subset $W \subseteq Y^{\prime}$ is called a base domain evenly covered by $p$, if $p^{-1}(W)$ is a disjoint union of open subsets $B_{l} \subseteq Y$

\footnotetext{
${ }^{10}$ Here " $\triangle_{\{p\}}$ " denotes the (local) pullback to $D^{*}$ under $p$ of the Laplace operator of the Euclidean metric on $\mathbb{C}^{m}$
} 
each of which is homeomorphic to $W$ (under $p$ ); as such $B_{l}$ will also be referred to as a covering sheet of $p$ lying evenly over $W$ (relative to $p$ ).

If $p: D \rightarrow \mathbb{C}^{m}$ is a light mapping ${ }^{11}$, then there exists an open connected neighborhood $U_{a} \Subset D$ of each $a \in D$, called a pseudoball at $a([21, \S 2])$ such that, when restricted to the regular part $\hat{U}_{a}:=U_{a} \backslash p^{-1}\left(\Delta^{\prime}\right)$ ( $\Delta$ being the branch locus), the map $p_{a}=p: \hat{U}_{a} \rightarrow U^{\prime} \backslash \Delta^{\prime}$ is an unramified finite holomorphic covering, where $U^{\prime}:=\mathbb{B}_{\left[a^{\prime}\right]}(r)$ is an open ball in $\mathbb{C}^{m}$ with center $a^{\prime}$ (see [1, $\S 2]$ ). The sheet number of $p_{a}$ is equal to $\nu_{p}(a)$, the multiplicity of $p$ at (the center) $a$ ([ibid., $\S 2]$ ). Thus at each point $z^{\prime} \in U^{\prime} \backslash \Delta^{\prime}$ there is an open ball $W^{z^{\prime}} \Subset U^{\prime} \backslash \Delta^{\prime}$ (of radius $<1$ ) (here $z^{\prime}$ will be denoted by $z_{k}^{\prime}$ and $W^{z^{\prime}}$ by $W^{k}$ for reasons to become clear later), which is a base domain evenly covered by $p_{a}$ with covering sheets $B^{\ell}=B_{l}^{z^{\prime}} \subseteq D, l=1, \cdots, l(k)$, over $W^{k}$, each being (necessarily) biholomorphic to $W^{k}$ under $p$. Here the number $l(k)$ is equal to $\nu_{p}(a)$ (for each $z_{k}^{\prime}$ ) and $B_{l}^{k}$ has compact closure in $\hat{U}_{a}$.

Definition 4.1. An admissible covering of a compact subset $\mathcal{K}$ of $D^{*}$ is an open covering of $\mathcal{K}$ consisting of open subsets $B^{\ell} \subseteq D^{*}$ with $\ell$ varying in a finite range such that each $B^{\ell}$ is equal to some $B_{l}^{k}$ (namely some $B_{l}^{z_{k}^{\prime}}$ ) with $k$ in a finite range and $l \in \mathbb{Z}[1, l(k)]$; moreover, for each fixed $k$ the $B_{l}^{k}$ (with varying l) lie evenly (relative to $p$ ) over a base domain $W^{k}:=W^{z_{k}^{\prime}}$ contained in some open ball $\hat{W}^{k}:=\hat{W}^{z_{k}^{\prime}} \subset \mathbb{C}^{m}$ of volume $<1$.

Note that every bounded domain $D$ in $\mathbb{C}^{m}$ admits an admissible covering (via a dilatation of the identity map). Also, if $U_{a}$ is a pseudoball contained in $D$, then every compact subset $\mathcal{K}$ of $\hat{U}_{a}$ admits an admissible covering.

The set $D^{*}$ being $\sigma$-compact, one can write $D^{*}$ as a union of an exhausting (increasing) sequence $\left\{\mathcal{K}_{j}\right\}$ of compact subsets. Choose a $\mathscr{C}^{\infty}$-partition of unity $\left\{\beta^{j, \ell}\right\}_{1 \leq \ell \leq N_{j}}$ on $\mathcal{K}_{j}$ subordinate to an admissible covering $\left\{B^{j, \ell}\right\}$ for $\mathcal{K}_{j}$. One has, for each $f \in \mathscr{C}^{\infty, c}(D)$, setting $f_{\ell}^{\{j\}}:=\left(\beta^{j, \ell}\right)^{\frac{1}{2}} f$ (the $\ell$-th partitioned function of $f$ relative to $\left.\left\{B^{j, \ell}\right\}\right)$,

$$
\int_{K_{j}}|f|^{2} d \tilde{v} \leq \sum_{\ell=1}^{N_{j}} \int_{D^{*}}\left|f_{\ell}^{\{j\}}\right|^{2} d \tilde{v} .
$$

A Riemann subdomain $(D, p)$ in $Y$ is said to be finitely quasiregular if (i) each compact subset $\mathcal{K}$ of $D^{*}$ admits a (finite) admissible covering $\left\{B_{l}^{k}\right\}$ with (corresponding) base domains $W^{k}$ with $\mathscr{C}^{1}$-boundary contained in some open ball of finite radius in $\mathbb{C}^{m}$; and (ii) the family $\left\{W^{k}\right\}$ has finite cardinality $\mathfrak{K}$ which depends only on $D$. If further $D$ is unramified, then $(D, p)$ is said to be finitely regular.

The above definition of "finitely quasiregular domain" is equivalent to the following: " $(D, p)$ is finitely quasiregular if the regular part $D^{*}$ is a finite union of local covering sheets $B^{\{\ell\}}, \ell=$

\footnotetext{
${ }^{11}$ A holommorphic mapping $f: Y \rightarrow Y^{\prime}$ (between complex spaces) is light on $D \subseteq Y$ if for each $a \in$ $D, \operatorname{dim}_{a} f^{-1}(f(a))=0$.
} 
$1, \cdots, \mathcal{N} "$. For, the above definition implies that, given any (increasing) sequence $\left\{\mathcal{K}_{j}\right\}$ of compact exhaustion of $D^{*}$, the index sequence $\left\{N_{j}\right\}$ (with $N_{j}$ arising from a corresponding $\mathcal{K}_{j}$ ) can be replaced by a finite sequence $\{1, \cdots, \mathcal{N}\}$ with $\mathcal{N}=l(1)+\cdots+l(\mathfrak{K})$. Thus $D^{*}$ is contained in (at most) $\mathcal{N}$ local covering sheets $B^{\{\ell\}}$. The converse assertion is trivially true.

Note that every bounded domain in $\mathbb{C}^{m}$ is finitely regular. Also, each relatively compact subset $D$ of the projective space $\mathbb{P}^{m}(\mathbb{C})$ contained in the open set $\mathbb{Q}^{\{s\}}, s \in \mathbb{Z}[1, m]$, is finitely regular relative to the Riemann covering $p=p^{[s]}$. For a further example, consider the complex space $Y=\left\{(z, w) \in \mathbb{C}^{2} \mid w^{2}=z^{3}\right\}$. Relative to the projection $(z, w) \mapsto z$ the open set $D:=\{(z, w) \in$ $\left.Y \mid\|z\| \leq r_{0}\right\}$ is a finitely quasiregular Riemann subdomain in $Y$ (with two covering sheets).

While for a general domain the Rellich Embedding Theorem may not be valid, the requirement that the domain be of Sobolev type is indeed somewhat restrictive. A characterization of the latter remains an open question. The following lemma is of some use:

Lemma 4.2. Every finitely quasiregular Riemann subdomain $(D, p)$ in $Y$ is of finite volume and of Sobolev type.

Proof. The regular part $D^{*}$ of a finitely quasiregular Riemann subdomain $(D, p)$ admits a finite covering by local covering sheets $B^{\ell}$ with $\ell$ varying in a finite range, say $\{1, \cdots, \mathcal{N}\}$. It is easy to show that $D$ has finite volume with respect to $p$. One can write $D^{*}$ as a union of an exhausting (increasing) sequence $\left\{\mathcal{K}_{j}\right\}$ of compact subsets. Each $\mathcal{K}_{j}$ admits a finite admissible covering $\left\{B^{j, \ell}\right\}_{1 \leq \ell \leq N_{j}}$. The Sobolev Embedding Inequality on a bounded Euclidean domain ([6, Theorem 6, p. 270] (applied to each $B^{j, \ell}$ ) implies that, for all $g \in \mathscr{C}^{\infty, c}(D)^{12}$,

$$
\begin{aligned}
\left(\|g\|_{L^{\alpha}(D)}\right)^{\alpha} & \leq \lim _{j \rightarrow \infty} \sum_{\ell=1}^{N_{j}} \int_{B^{j, \ell}}|g|^{\alpha} d \tilde{v}=\lim _{j \rightarrow \infty} \sum_{\ell=1}^{N_{j}}\left(\|g\|_{L^{\alpha}\left(B^{j, \ell}\right)}\right)^{\alpha} \\
& \leq\left(C_{B_{m}}\right)^{\alpha} \lim _{j \rightarrow \infty} \sum_{\ell=1}^{N_{j}}\left(\|g\|_{H_{1,(0,1, \cdots, 1)}^{1}\left(B^{j, \ell}\right)}\right)^{\alpha} \\
& \leq\left(C_{B_{m}}\right)^{\alpha} \lim _{j \rightarrow \infty} \sum_{\ell=1}^{N_{j}}\left(\|g\|_{H_{1,(0,1, \cdots, 1)}^{1}(D)}\right)^{\alpha}
\end{aligned}
$$

for some constant $C_{B_{m}}$ (independent of $D$ ). Since the local covering sheets $B^{j, \ell}$ can be selected from the finite set $\left\{B^{\ell}\right\}_{\ell=1, \cdots, \mathcal{N}}$, the above index $N_{j}$ can be replaced by $\mathcal{N}$. Thus

$$
\left(\|g\|_{L^{\alpha}(D)}\right)^{\alpha} \leq \text { Const. }\left(\|g\|_{H_{1,(0,1, \cdots, 1)}^{1}(D)}\right)^{\alpha} .
$$

The space $\mathscr{C}^{\infty, c}(D)$ being dense in $H_{1,(0,1, \cdots, 1), c}^{1}(D)$, each element $f$ therein can be approximated in the $L^{2}$-norm by a sequence $\left\{g_{n}\right\} \subset \mathscr{C}^{\infty, c}(D)$. Thus the Sobolev inequality (1.4) follows.

\footnotetext{
12 For clarity denote the Sobolev norm of $g \in H_{1,(0,1, \cdots, 1)}^{1}\left(B^{\ell}\right)$ by $\|g\|_{H_{1,(0,1, \cdots, 1)}^{1} .\left(B^{\ell}\right)}$ and similarly for $g \in$ $H_{1,(0,1, \cdots, 1), c}^{1}(D)$.
} 
Proof of Theorem. 1.1. For later reference, assume (unless otherwise mentioned) that $\mathfrak{h}$ denotes a general allowable weight as indicated in the beginning of $\S 1$. Let $\left\{f_{n}\right\}$ be a bounded sequence in $H_{\mu, \mathfrak{h}, c}^{1}(D)$. It contains a subsequence $\left\{f_{n_{k}}\right\}$ which converges weakly to some element $f \in$ $H_{\mu, \mathfrak{h}, c}^{1}(D)$. By considering $\left\{f_{n_{k}}-f\right\}$ it may be assumed that $\left\{f_{n_{k}}\right\} \subset\left\{f_{n}\right\}$ is a subsequence converging weakly to 0 . In the following for simplicity denote this subsequence by the same notation $\left\{f_{n}\right\}$. Since $\mathscr{C}^{\infty, c}(D)$ is dense in $H_{\mu, \mathfrak{h}, c}^{1}(D)$, each $f_{n}$ is the limit of a sequence $\left\{\phi_{l}^{n}\right\} \subset$ $\mathscr{C}^{\infty, c}(D)$ with respect to the \|\|$_{\mu, \mathfrak{h}}$-norm on $H_{\mu, \mathfrak{h}, c}^{1}(D)$. Thus, for each $n \in \mathbb{N}$ there exists an element $\phi^{\{n\}} \in \mathscr{C}^{\infty, c}(D)$ such that

$$
\left\|f_{n}-\phi^{\{n\}}\right\|_{\mu, \mathfrak{h}}<\frac{1}{n}
$$

Then for any given $\rho \in H_{\mu, \mathfrak{h}, c}^{1}(D)$,

$$
\left(\phi^{\{n\}}, \rho\right)_{\mu, \mathfrak{h}}=\left(\phi^{\{n\}}-f_{n}, \rho\right)_{\mu, \mathfrak{h}}+\left(f_{n}, \rho\right)_{\mu, \mathfrak{h}} \rightarrow 0, \quad n \rightarrow \infty
$$

namely $\phi^{\{n\}}$ tends to 0 weakly in $H_{\mu, \mathfrak{h}, c}^{1}(D)$. Since

$$
\left\|f_{n}-\phi^{\{n\}}\right\|_{L^{2}(D)} \leq \text { Const. }\left\|f_{n}-\phi^{\{n\}}\right\|_{\mu, \mathfrak{h}}
$$

to prove the Rellich Embedding Theorem it suffices to consider the case where $\left\{f_{n}\right\}$ lies in $\mathscr{C}^{\infty, c}(D)$ and converges weakly to 0 in $H_{\mu, \mathfrak{h}, c}^{1}(D)$ (in which case $\left\{f_{n}\right\}$ is uniformly bounded in $H_{\mu, \mathfrak{h}, c}^{1}(D)$ ). In the rest of the proof, assume that $(\mu, V)=(1,0)$. The goal is to show (possibly by passing to a subsequence) that $\lim _{n \rightarrow \infty}\left\|f_{n}\right\|_{L^{2}(D)}^{2}$ exists and equals zero. For this purpose it will be necessary to swap the order of the limits in the following relation

$$
\lim _{n \rightarrow \infty} \lim _{j \rightarrow \infty} \int_{K_{j}}\left|f_{n}\right|^{2} d \tilde{v}=\lim _{j \rightarrow \infty} \lim _{n \rightarrow \infty} \int_{K_{j}}\left|f_{n}\right|^{2} d \tilde{v}
$$

(where $\left\{K_{j}\right\}$ is an increasing exhausting sequence of compact subsets of $D^{*}$ ). This can be justified by verifying two conditions: (a) the sequence of functions

$$
\Phi_{j}(n):=\int_{K_{j}}\left|f_{n}\right|^{2} d \tilde{v} \text { converges to } \Phi(n):=\lim _{j \rightarrow \infty} \int_{K_{j}}\left|f_{n}\right|^{2} d \tilde{v}
$$

(as $j \rightarrow \infty$ ) uniformly in $n$, and (b) for each fixed $j$ the limit " $\lim _{n \rightarrow \infty} \int_{K_{j}}\left|f_{n}\right|^{2} d \tilde{v}$ " exists (in fact, equals 0). More explicitly, the assertion (a) amounts to showing that, for each $\epsilon>0, \exists J_{\epsilon}$ and $N_{\epsilon} \in \mathbb{N}$ such that

$$
\int_{\left(K_{J_{\epsilon}}\right)^{c}}\left|f_{n}\right|^{2} d \tilde{v} \leq \epsilon, \quad \forall n \geq N_{\epsilon} .
$$

To prove this condition, set $\frac{1}{q^{\prime}}:=\frac{2}{\alpha}$ and $\frac{1}{p^{\prime}}:=1-\frac{1}{q^{\prime}}$. Then by Hölder's inequality (with $f=1$ and $g=\left|f_{n}\right|^{2}$ ) the left-hand side of the above inequality is dominated by

$$
\left.\int_{\left(K_{J_{\epsilon}}\right)^{c}}|1 \cdot| f_{n}\right|^{2} \mid d \tilde{v} \leq\left(\int_{\left(K_{J_{\epsilon}}\right)^{c}} 1^{p^{\prime}} d \tilde{v}\right)^{\frac{1}{p^{\prime}}}\left(\int_{\left(K_{J_{\epsilon}}\right)^{c}}\left(\left|f_{n}\right|^{2}\right)^{q^{\prime}} d \tilde{v}\right)^{\frac{1}{q^{\prime}}} .
$$


Since $\left(\left|f_{n}\right|^{2}\right)^{q^{\prime}}=\left|f_{n}\right|^{\alpha}$, and

$$
\left(\left\|\left|f_{n}\right|^{2}\right\|\right)_{L^{q^{\prime}}\left(\left(K_{J_{\epsilon}}\right)^{c}\right)}=\left(\int_{\left(K_{J_{\epsilon}}\right)^{c}}\left|f_{n}\right|^{\alpha} d \tilde{v}\right)^{\frac{2}{\alpha}}=\left(\left\|f_{n}\right\|_{L^{\alpha}\left(\left(K_{J_{\epsilon}}\right)^{c}\right)}\right)^{2},
$$

the inequality (4.4) becomes

$$
\int_{\left(K_{J_{\epsilon}}\right)^{c}}\left|f_{n}\right|^{2} d \tilde{v} \leq\left|\operatorname{vol}\left(\left(K_{J_{\epsilon}}\right)^{c}\right)\right|^{1-\frac{2}{\alpha}}\left\|f_{n}\right\|_{L^{\alpha}\left(\left(K_{J_{\epsilon}}\right)^{c}\right)}^{2}
$$

Therefore (if $\alpha$ is chosen to satisfy the Sobolev Inequality (1.4)) the second factor on the right-hand side is uniformly bounded, hence one has

$$
\int_{\left(K_{J_{\epsilon}}\right)^{c}}\left|f_{n}\right|^{2} d \tilde{v} \leq \text { Const. }\left|\operatorname{vol}\left(\left(K_{J_{\epsilon}}\right)^{c}\right)\right|^{1-\frac{2}{\alpha}} .
$$

Finally, since $(D, p)$ has finite volume, by letting $J_{\epsilon} \rightarrow \infty$ one has $\operatorname{vol}\left(\left(K_{J_{\epsilon}}\right)^{c}\right) \rightarrow 0$. Thus the condition for uniform convergence (4.3) follows.

By choice of an open covering of $K_{j}$ by local covering sheets $B^{\ell}$ (with notations as in Definition 4.1) each of which is contained in a fixed open neighborhood $D_{j} \Subset D^{*}$ (the sheets $B^{\ell}$ and $D_{j}$ being dependent only on $j$ ), all the terms $f_{n, \ell}^{\{j\}}$ have support contained in $\overline{D_{j}}$. Denoting by $\hat{f}_{n, \ell}^{\{j\}}$ the direct image of $f_{n, \ell}^{\{j\}} \mid B^{\ell}$ (under the biholomorphic map $p: B^{\ell} \rightarrow \mathcal{B}^{\ell}=p\left(B^{\ell}\right.$ ), the resulting family $\left\{\hat{f}_{n, \ell}^{\{j\}}\right\}$ is uniformly bounded in $H_{\mu, \mathfrak{h}, c}^{1}\left(\mathcal{B}^{\ell}\right)$ with respect to $n, \ell$. Inscribe $\mathcal{B}^{\ell}$ in a cube $C$ (independent of $\ell$, and it may be assumed that $C \subset \mathbb{B}(1)$ by rescaling and translation). Every element $\hat{\rho} \in \mathscr{C}^{\infty, c}\left(\mathcal{B}^{\ell}\right)$ can be extended to $C$ trivially (by setting $\hat{\rho}$ to be zero off $\mathcal{B}^{\ell}$ ).

The weak convergence of $\left\{f_{n}\right\}$ (to 0$)$ in $H_{\mu, \mathfrak{h}, c}^{1}\left(B^{\ell}\right)$ implies that of $f_{n, \ell}^{\{j\}}$, hence $\hat{f}_{n, \ell}^{\{j\}}$ converges weakly to 0 in $H_{1, \mathfrak{h}, c}^{1}\left(\mathcal{B}^{\ell}\right)$. The preceding discussion shows that the compactness of the embedding " $H_{1, \mathfrak{h}, c}^{1}(D) \hookrightarrow L^{2}(D)$ " is a consequence of the following "claim":

If $\left\{\hat{f}_{n, \ell}^{\{j\}}\right\}$ is a sequence (indexed by $n$ ) in $H_{1,\left(0, \mathfrak{h}^{\prime}\right), c}^{1}\left(\mathcal{B}^{\ell}\right)$ which converges weakly to 0 in $H_{1,\left(0, \mathfrak{h}^{\prime}\right), c}^{1}\left(\mathcal{B}^{\ell}\right)$, the $\mathfrak{h}^{\prime}$ being an allowable constant $2 m$-tuple, then

$$
\lim _{n \rightarrow \infty} \int_{D^{*}}\left|f_{n, \ell}^{\{j\}}\right|^{2} d \tilde{v}=0
$$

Upon taking $\mathfrak{h}=(0, \underset{=}{h})$ with $\stackrel{h}{=}=(1, \cdots, 1)$, this is an immediate consequence of the Rellich Embedding Theorem for a bounded Euclidean domain (here the open ball $\mathcal{B}^{\ell}$ ). This proves the assertion (b). By interchanging the " $n$-" and the " $j$-limit" and making use of the relations (4.2) and (4.1), one has

$$
\begin{aligned}
\lim _{n \rightarrow \infty} \lim _{j \rightarrow \infty} \int_{K_{j}}\left|f_{n}\right|^{2} d \tilde{v} & =\lim _{j \rightarrow \infty} \lim _{n \rightarrow \infty} \int_{K_{j}}\left|f_{n}\right|^{2} d \tilde{v} \\
& \leq \lim _{j \rightarrow \infty} \lim _{n \rightarrow \infty} \sum_{\ell=1}^{N_{j}} \int_{D^{*}}\left|f_{n, \ell}^{\{j\}}\right|^{2} d \tilde{v}=0 .
\end{aligned}
$$


Consequently Theorem 1.1 is proved in this case. Let $\mathfrak{h}^{\prime}=\left(c_{1}, \cdots, c_{2 m}\right)$ be a $2 m$-tuple of positive constants. Define $\hat{p}=\hat{p}_{\left\{\mathfrak{h}^{\prime}\right\}}=\left(\hat{p}_{1}, \cdots, \hat{p}_{m}\right): D \rightarrow \mathbb{C}^{m}$ by setting

$$
\hat{p}_{k}(z):=\hat{x}_{k}+i \hat{y}_{k}=\left(c_{2 k-1}\right)^{-1 / 2} \tilde{x}_{k}+i\left(c_{2 k}\right)^{-1 / 2} \tilde{y}_{k}, \quad 1 \leq k \leq m,
$$

Then the volume element

$$
d \hat{v}\left(\mathfrak{h}^{\prime}\right):=\hat{p}^{*}(d v)=c_{1}^{-1 / 2} \cdots c_{2 m}^{-1 / 2} d \tilde{v} .
$$

It is easy to show that the norm of an element $f$ of $\hat{H}_{1,\{0,1, \cdots, 1\}, c}^{1}(D)$, that is, the space $H_{1,\{0,1, \cdots, 1\}, c}^{1}(D)$ defined w. r. t. $\left(D, \hat{p}_{\left\{\mathfrak{h}^{\prime}\right\}}\right)$ with $\mathfrak{h}^{\prime}=(1, \cdots, 1)$, is equivalent to the norm of $f$ in $H_{1,\left\{0, c_{1}, \cdots, c_{2 m}\right\}, c}^{1}(D)$ w. r. t. $(D, p)$. Hence the Sobolev space $\hat{H}_{1,\{0,1, \cdots, 1\}, c}^{1}(D)$ is the same as $H_{1,\left\{0, c_{1}, \cdots, c_{2 m}\right\}, c}^{1}(D)$. Since the embedding " $\hat{H}_{1,\{0,1, \cdots, 1\}, c}^{1}(D) \hookrightarrow L^{2}(D)$ " is compact, the same is true for the embedding " $H_{1,\left\{0, c_{1}, \cdots, c_{2 m}\right\}, c}^{1}(D) \hookrightarrow L^{2}(D)$ ". This completes the proof Theorem 1.1.

A Riemann subdomain $(D, p)$ in $Y$ is said to define a (distinguished) étale covering ${ }^{13}$ (of $p\left(D^{*}\right)$ ) if (i) each compact subset $\mathcal{K}$ of $D^{*}$ admits a (finite) admissible covering $\left\{B_{l}^{k}\right\}$ (with corresponding base domains $W^{k}$ ); and (ii) the family $\left\{W^{k}\right\}$ is pair-wise disjoint. Note that every bounded domain $D$ in $\mathbb{C}^{m}$ defines a distinguished étale covering (via a rescaled identity map). As another example, consider the $m$-dimensional projective space $\mathbb{P}^{m}(\mathbb{C})$ and let $\mathbb{Q}^{\{s\}}:=\{a=$ $\left.\left[a_{0}, \cdots, a_{m}\right] \mid a_{s} \neq 0\right\}$, where $s \in \mathbb{Z}[1, m]$. Then $\mathbb{Q}^{\{s\}}$ can be regarded as an open Riemann subdomain in $\mathbb{P}^{m}(\mathbb{C})$ relative to the $s$-th canonical coordinate map $p^{[s]}: \mathbb{Q}^{\{s\}} \rightarrow \mathbb{C}^{m}$ given by

$$
p^{[s]}: a \mapsto\left(\frac{a_{0}}{a_{s}}, \cdots, \frac{\widehat{a_{s}}}{a_{s}}, \cdots, \frac{a_{m}}{a_{s}}\right)
$$

(here "ح" denotes omission). Clearly every relatively compact subset $D \Subset \mathbb{Q}^{\{s\}}$ defines a distinguished étale covering via the Riemann covering $p=p^{[s]}$.

Proof of Theorem 1.2. Let $\mathfrak{h}^{\prime}=\left(h_{1}, \cdots, h_{2 m}\right)$ be any allowable weight on $D$. To prove the Poincaré inequality (1.3), it suffices to verify it for all elements $g \in \mathscr{C}^{\infty, c}(D)$. For, given $f \in$ $H_{1, \mathfrak{\mathfrak { h } ^ { \prime }}, c}^{1}(D)$, there exists a sequence $\left\{g_{n}\right\}$ in $\mathscr{C}^{\infty, c}(D)$ converging to $f$ in $H_{1, \mathfrak{\mathfrak { h } ^ { \prime }}, c}^{1}(D)$, that is,

$$
\left(\left\|f-g_{n}\right\|_{L^{2}(D)}^{2}+\left[f-g_{n}, f-g_{n}\right]_{D, \mathfrak{h}^{\prime}}\right)^{\frac{1}{2}} \rightarrow 0 \quad \text { as } n \rightarrow \infty
$$

If

$$
\left\|g_{n}\right\|_{L^{2}(D)} \leq C_{D, \mathfrak{h}^{\prime}}\left[g_{n}, g_{n}\right]_{D, \mathfrak{h}^{\prime}}^{\frac{1}{2}}, \quad \forall n \geq 1
$$

then the limit relation (4.5) implies that inequality (1.3) holds. Thus the above claim holds true. Assume that $(D, p)$ defines an étale covering $\left\{B_{l}^{k}\right\}$ of $p\left(D^{*}\right)$. Let $g \in \mathscr{C}^{1, c}(D)$ and $K:=\operatorname{Spt}(g)$. The regular part $D^{*}$ being $\sigma$-compact, the set $K \cap D^{*}$ admits an exhausting sequence of increasing compact subsets $\left\{\mathcal{K}_{j}\right\}$ of $K \cap D^{*}$, each of which is contained in a finite union of covering sheets

\footnotetext{
${ }^{13}$ See Barlet [2, p. 110] for a closely related notion of "revêtement analytique étale".
} 
$B^{\ell}$ (namely some $B_{l}^{k}$ as above). Choose a $C^{\infty}$-partition of unity $\left\{\beta^{j, \ell}\right\}_{1 \leq \ell \leq N^{j}}$ on $\mathcal{K}_{j}$ subordinate to such a covering $\left\{B^{\ell}\right\}_{\ell=1, \cdots, N^{j}}$ (with monotonically increasing index $N^{j}$ ). Assume (without loss of generality) that $\sum_{1 \leq \ell \leq N^{j}} \beta^{j, \ell}=1$ on $D_{j}:=\cup\left\{B^{\ell} \mid 1 \leq \ell \leq N^{j}\right\}$. Let $\hat{g}^{\{j, \ell\}}: \mathcal{B}^{\ell}:=p\left(B^{\ell}\right) \rightarrow \mathbb{C}$ be the direct image of $g^{\{j, \ell\}}:=\beta^{j, \ell} g$ (under the map $p$ ). Assume now that $D$ is unramified. Then $D=D^{*}$, hence the $\left\{\mathcal{K}_{j}\right\}$ can be chosen to be a finite sequence with $K_{j}=K$ for large enough $j$. Then one has

$$
\begin{aligned}
\int_{D}|g|^{2} d \tilde{v} & =\int_{D_{j}}|g|^{2} d \tilde{v}=\int_{D_{j}} \sum_{\ell}\left(\beta^{j, \ell}\right)^{2}|g|^{2} d \tilde{v} \\
& =\int_{D_{j}} \sum_{\ell}\left(\beta^{j, \ell}\right)^{2}|g|^{2} d \tilde{v}+2 \int_{D_{j}}\left(\sum_{\ell \neq \ell^{\prime}} \beta^{j, \ell} \beta^{j, \ell^{\prime}}\right)|g|^{2} d \tilde{v} \\
& =\sum_{\ell} \int_{\mathcal{B}^{\ell}}\left|\hat{g}^{\{j, \ell\}}\right|^{2} d v
\end{aligned}
$$

where the added integral (in the second equality) vanishes since the base domains of the covering sheets $B^{\ell}$ are pair-wise disjoint. Note that $g=\sum_{1 \leq \mathfrak{l} \leq N^{j}} \beta^{j,\{\mathfrak{l}\}}$ on $D_{j}$, By resorting to the Poincaré inequality for the Euclidean unit ball, one has ${ }^{14}$, for a given $g \in \mathscr{C}^{\infty, c}(D)$,

$$
\begin{aligned}
\frac{\mathfrak{h}_{D}}{\left(\mathcal{P}_{\mathbb{B}}\right)^{2}} \sum_{\ell} \int_{\mathcal{B}^{\ell}}\left|\hat{g}^{\{j, \ell\}}\right|^{2} d v & \leq \mathfrak{h}_{D} \sum_{\ell} \int_{\mathcal{B}^{\ell}}\left|\nabla \hat{g}^{\{j, \ell\}}\right|^{2} d v \leq \int_{D_{j}} \sum_{\ell} \sum_{\lambda=1}^{2 m} h_{\lambda}\left|\partial_{\lambda}\left(g^{\{j, \ell\}}\right)\right|^{2} d \tilde{v} \\
& =\int_{D_{j}} \sum_{\lambda=1}^{2 m} h_{\lambda} \sum_{\ell, \ell^{\prime}} \partial_{\lambda}\left(g^{\{j, \ell\}}\right) \partial_{\lambda}\left(\bar{g}^{\left\{j, \ell^{\prime}\right\}}\right) d \tilde{v} \\
& =\int_{D_{j}} \sum_{\lambda=1}^{2 m} h_{\lambda}\left(\sum_{\ell} \partial_{\lambda}\left(g^{\{j, \ell\}}\right)\right)\left(\sum_{\ell^{\prime}} \partial_{\lambda}\left(\bar{g}^{\left\{j, \ell^{\prime}\right\}}\right)\right) d \tilde{v} \\
& =\int_{D_{j}} \sum_{\lambda=1}^{2 m} h_{\lambda} \partial_{\lambda}\left(\sum_{\ell} g^{\{j, \ell\}}\right) \partial_{\lambda}\left(\sum_{\ell^{\prime}} \bar{g}^{\left\{j, \ell^{\prime}\right\}}\right) d \tilde{v} \\
& =[g, g]_{D_{j},\left(h_{1}, \cdots, h_{2 m}\right)}=[g, g]_{D,\left(h_{1}, \cdots, h_{2 m}\right)},
\end{aligned}
$$

where $\mathfrak{h}_{D}=\min \left\{\operatorname{essinf}_{D}\left(h_{j}\right) \mid 1 \leq j \leq 2 m\right\}>0$, and $\mathcal{P}_{\mathbb{B}}$ denotes the Poincaré's constant for the unit ball. Consequently the Poincaré inequality (1.3) follows.

Proposition 4.3 (Generalized Poincaré-Wirtinger Inequality $\left.{ }^{15}\right)$. Assume that $(D, p)$ is a Riemann subdomain such that, with respect to a continuous allowable weight $\mathfrak{h}^{\prime}$ on $D$, the RellichKondrachov embedding property holds: $\underset{1, \underset{\mathfrak{h}^{\prime}}{\rightarrow}}{H_{(}^{1}}(D) \hookrightarrow L^{2}(D)$ is compact. Then there exists a constant $C_{D}$ such that

$$
\left\|f-\frac{1}{\operatorname{vol}(D)} \int_{D} f d \tilde{v}\right\|_{L^{2}(D)} \leq C_{D}[f, f]_{D, \mathfrak{h}^{\prime}}^{\frac{1}{2}}, \quad \forall f \in \underset{1, \underset{\mathfrak{h}^{\prime}}{\rightarrow}}{\stackrel{1}{\longrightarrow}}(D) .
$$

\footnotetext{
${ }^{14} \mathrm{On}$ the right-side of the following, in the integral of the second inequality a sum of terms "const $\int_{D_{j}} \sum_{\lambda} h_{\lambda} \sum_{\ell \neq \ell^{\prime}} \partial_{\lambda}\left(g^{\{j, \ell\}}\right) \partial_{\lambda}\left(\bar{g}^{\left\{j, \ell^{\prime}\right\}}\right) d \tilde{v}$ " may be added, since it vanishes owing to the fact that the base domains $W^{k}$ are pair-wise disjoint.

${ }^{15}$ A version of this inequality is presented in Deny-Lions [5, (5.5), p.329].
} 
The proof (by way of reductio ad absurdum) is omitted.

Definition 4.4. Let $D \subseteq Y$ be a Riemann subdomain with $d D \neq \emptyset, f \in \operatorname{Lip}(\partial D ; \mathbb{C})^{16}$, and $\varphi \in \mathscr{C}_{2 m}^{0}(D \backslash \mathscr{W})$, where $\mathscr{W}$ is a thin ${ }^{17}$ analytic subset of $D$. A weak solution of the Dirichlet problem for the Poisson equation:

$$
d d^{c} u \wedge v_{p}^{m-1}=\varphi \quad \text { in } D \backslash \mathscr{W}, \quad u|d D=f| d D
$$

is an element $u=w \in H_{1,(0,1, \cdots, 1)}^{1}(D)$ such that $w \equiv f \bmod \left(H_{1,(0,1, \cdots, 1), c}^{1}(D)\right)$, and

$$
[w, v]_{D,(1, \cdots, 1)}=(\varphi, v)_{D}, \quad \forall v \in H_{1,(0,1, \cdots, 1), c}^{1}(D) .
$$

Corollary 4.5. Let $D \subseteq Y$ be a Riemann subdomain with $d D \neq \emptyset$. Assume that $(D, p)$ has the Poincaré property relative to the unit $2 m$-weight $(1, \cdots, 1)$. Then for any $f \in \operatorname{Lip}(\partial D ; \mathbb{C})$ and $\varphi \in \mathscr{C}_{2 m}^{0}(D \backslash \mathscr{W}), \mathscr{W}$ being thin analytic in $D$, the Dirichlet problem (4.7) admits a weak solution $w \in H_{1,(0,1, \cdots, 1)}^{1}(D)$.

Proof. Consider the linear mapping $T: H_{1,(0,1, \cdots, 1), c}^{1}(D) \rightarrow \mathbb{C}$ defined by

$$
T(v):=(\varphi, \bar{v})_{D}-[\tilde{f}, v]_{D,(1, \cdots, 1)}, \quad \forall v \in H_{1,(0,1, \cdots, 1), c}^{1}(D) .
$$

where $\tilde{f}$ is a Lipschitzian extension of $f$ to a neighborhood of $\bar{D}$. Then the Poincare inequality (1.3) (with $\mathfrak{h}^{\prime}=(1, \cdots, 1)$ ) implies that the Riesz representation theorem is applicable to the operator $T$. Therefore there exists an element $w_{0} \in H_{1,(0,1, \cdots, 1), c}^{1}(D)$ such that

$$
T(v)=\left[w_{0}, v\right]_{D,(1, \cdots, 1)}, \quad \forall v \in H_{1,(0,1, \cdots, 1), c}^{1}(D) .
$$

Then $w:=w_{0}+\tilde{f} \in H_{1,(0,1, \cdots, 1)}^{1}(D)$ is a weak solution to the Dirichlet problem (4.7).

\section{The resolvent map for an inhomogeneous Dirichlet problem}

Let $\mathfrak{h}^{\prime}$ be an allowable $2 m$-weight on $D$ and $H_{\substack{1, \mathfrak{h}^{\prime}, c \\ \rightarrow}}^{1}(D)$ be equipped with the Dirichlet norm (defined by (1.2)). For fixed $f \in L^{2}(D)$, applying the Riesz's representation theorem to the bounded anti-linear functional $v \mapsto(f, \bar{v})_{D}$ on $H_{1, \mathfrak{h ^ { \prime }}, c}^{1}(D)$, yields an element $w \in H_{1, \mathfrak{h}}^{1}, c(D)$ such that

$$
(f, \bar{v})_{D}=[w, v]_{D, \mathfrak{h}^{\prime}}, \quad \forall v \in H_{1, \mathfrak{h}^{\prime}, c}^{1}(D) .
$$

\footnotetext{
${ }^{16} f \in \operatorname{Lip}(\partial D ; \mathbb{C})$ means that $f$ is (locally) Lipschitzian in a neighborhood of $\partial D$. As such it admits a Lipschitzian extension $\tilde{f}$ to a neighborhood of $\bar{D}$ by invoking a partition of unity.

${ }^{17}$ A subset $T$ of an $m$-dimensional complex space $Y$ is thin, if at each point $a \in T$ there is an analytic subset $A$ of dimension $<m$ in an open neighborhood $U \subseteq Y$ of $a$ such that $T \cap U \subseteq A$.
} 
The association " $f \mapsto \mathscr{R}_{D, \mathfrak{h}^{\prime}} f=w$ " defines a continuous linear mapping $\mathscr{R}_{D, \mathfrak{h}^{\prime}}: L^{2}(D) \rightarrow L^{2}(D)$ with image in $H_{1, \mathfrak{\mathfrak { h } ^ { \prime }}, c}^{1}(D)$ satisfying the equation (1.7).

Assume now that $(D, p)$ has the Poincaré property relative to a bounded, $\mathscr{C}^{\infty}$ allowable $2 m$ weight $\mathfrak{h}^{\prime}{ }^{18}$. Then the Poincaré inequality (1.3) implies that the Sobolev spaces $\underset{1, \underset{\mathfrak{h}^{\prime}, c}{\rightarrow}}{\rightarrow}(D)$ and $H_{0, \underset{\mathfrak{h}^{\prime}}{\rightarrow}, c}^{1}(D)$ are defined by equivalent norms, hence can be naturally identified with each other. The mapping $G_{0, \mathfrak{\mathfrak { h } ^ { \prime }}}: L^{2}(D) \rightarrow H_{1, \mathfrak{\mathfrak { h } ^ { \prime }}, c}^{1}(D)$ (the latter being identified with $H_{0, \mathfrak{h}}^{1} \underset{\rightarrow}{\rightarrow}, c(D)$ ) is continuous, linear and satisfies, for each fixed $\psi \in L^{2}(D)$, the equation

$$
(\psi, \bar{v})_{D}=\left\langle G_{0, \underset{\mathfrak{h}^{\prime}}{\rightarrow}}^{\rightarrow} \psi, v\right\rangle_{0,\left(0, \mathfrak{h}^{\prime}\right)}=\left[G_{0, \mathfrak{h}^{\prime}}^{\rightarrow} \psi, v\right]_{D, \mathfrak{h}^{\prime}}, \quad \forall v \in H_{1, \mathfrak{h}^{\prime}, c}^{1},(D) .
$$

Hence it follows that $\mathscr{R}_{D, \mathfrak{h}^{\prime}}=G_{0, \mathfrak{h}^{\prime}}: L^{2}(D) \rightarrow H_{1, \mathfrak{h}^{\prime}, c}^{1}(D)$. Also, since the mapping $G_{0, \mathfrak{h}^{\prime}}$ is injective, so is the mapping $\mathscr{R}_{D, \mathfrak{h}^{\prime}}$. Thus $\mathscr{R}_{D, \mathfrak{h}^{\prime}}$ can (justifiably) be called the resolvent map for the differential operator defined by the left-side of the equation (1.8).

Proof of Theorem 1.3. It follows from the hermitian symmetry of the Dirichlet product that the mapping $\mathscr{R}_{D, \mathfrak{h}^{\prime}}: L^{2}(D) \rightarrow L^{2}(D)$ is self-adjoint. In the rest of this proof write "( $\left.\mu, \mathfrak{h}\right)$ " for the pair $(1,\{0,1, \cdots, 1\})$. The mapping $\mathscr{R}_{D, \mathfrak{h}^{\prime}}$ is expressible as a composition of the (restricted) mapping $\tilde{\mathscr{R}}_{D, \mathfrak{h}^{\prime}}: L^{2}(D) \rightarrow H_{\mu, \mathfrak{h}, c}^{1}(D)$ and the compact Rellich embedding $\mathfrak{i}: H_{\mu, \mathfrak{h}, c}^{1}(D) \hookrightarrow L^{2}(D)$. Consequently $\mathscr{R}_{D, \mathfrak{h}^{\prime}}=\mathfrak{i} \circ \tilde{\mathscr{R}}_{D, \mathfrak{h}^{\prime}}$ is compact.

Remark 5.1. The above (same) proof of the embedding Theorem 1.3 yields the following: Assume that the embedding $H_{\mu, \mathfrak{h}, c}^{1}(D) \hookrightarrow L^{2}(D)$ is compact for a (given) allowable weight $\mathfrak{h}$ for $(D, \mu)$. Then there is defined a compact mapping $\mathscr{R}_{D, \mathfrak{h}^{\prime}}^{\mu}: L^{2}(D) \rightarrow L^{2}(D)$ (in a way similar to that for $\left.\mathscr{R}_{D, \mathfrak{h}^{\prime}}=\mathscr{R}_{D, \mathfrak{h}^{\prime}}^{1}: L^{2}(D) \rightarrow L^{2}(D)\right)$.

\section{Solution of an Eigenvalue Problem}

Theorem 6.1. Assume that $(D, p)$ is a Rellich subdomain with respect to $\mathfrak{h}^{\prime}$ (the latter being a given allowable $2 m$-weight). Then:

(a) there exists a non-decreasing, unbounded sequence $\left\{\lambda_{j}\right\}$ of positive real numbers such that the operator equation

$$
[u, v]_{D, \mathfrak{h}^{\prime}}=\lambda(u, \bar{v})_{D} \quad \forall v \in \mathscr{C}^{\infty, c}(D), \quad u \mid d D=0,
$$

admits a nontrivial solution $u \in H_{\substack{1, \mathfrak{h}^{\prime}, c \\ \rightarrow}}^{1}(D)$ precisely when $\lambda$ is a member of the countable set $\left\{\lambda_{j}\right\}$;

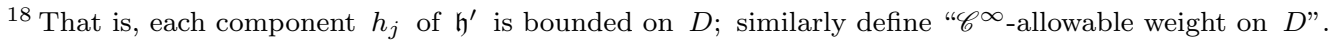


(b) there exists an orthonormal basis $\left\{\psi_{k}\right\}$ of $H_{1, \mathfrak{h}^{\prime}, c}^{1}(D)$, consisting of eigenfunctions of $\mathscr{R}_{D, \mathfrak{h}^{\prime}}$, which is complete in both $L^{2}(D)$ and $H_{\substack{1, \mathfrak{h}^{\prime}, c \\ \rightarrow}}^{1}(\vec{D})$; furthermore, if $\mathfrak{h}$ is of class $\mathscr{C}^{\infty}$ on $D^{*}$, then each $\psi_{k} \in H_{\substack{1, \mathfrak{h}^{\prime}, c \\ \text {, }}}^{1}(D) \cap \mathscr{C}^{\infty}\left(D^{*}\right)$.

Proof. By the Remark 5.1, the mapping $\mathscr{R}_{D, \mathfrak{h}^{\prime}}: L^{2}(D) \rightarrow L^{2}(D)$ is compact, and self-adjoint. Hence it has real eigenvalues $\left\{\beta_{j}\right\}_{j \in \mathbb{N}}$ of finite multiplicity (equal to the dimension of the eigenspace $\left.E_{j}:=\operatorname{ker}\left(\mathscr{R}_{D, \mathfrak{h}^{\prime}}-\beta_{j} I\right)\right)$ may be arranged as a sequence $\left|\beta_{1}\right| \geq\left|\beta_{2}\right| \geq \cdots$, with no point of accumulation except possibly the origin. The members of (distinct) $E_{j}$ and $E_{j^{\prime}}$ are mutually orthogonal. Since the mapping $\mathscr{R}_{D, \mathfrak{h}^{\prime}}$ takes values in $H_{\substack{1, \mathfrak{h}^{\prime}, c \\ \rightarrow}}^{1}(D)$, for each $j=1,2 \cdots$ and $\phi_{j} \in E_{j}$, the relation

$$
\mathscr{R}_{D, \mathfrak{h}^{\prime}} \phi_{j}=\beta_{j} \phi_{j}
$$

holds and implies that $\phi_{j}$ belongs to $H_{\substack{1, \mathfrak{h}^{\prime}, c \\ \rightarrow}}^{1}(D)$. Moreover,

$$
\beta_{j}\left[\phi_{j}, \phi_{k}\right]_{D, \mathfrak{h}^{\prime}}=\left[\mathscr{R}_{D, \mathfrak{h}^{\prime}} \phi_{j}, \phi_{k}\right]_{D, \mathfrak{h}^{\prime}}=\left(\phi_{j}, \bar{\phi}_{k}\right)_{D}, \quad \forall j, k=1,2, \cdots .
$$

This implies that each $\beta_{j}>0$. By orthonormalizing a basis of each $E_{j}$ and taking their union, one obtains an orthonormal basis $\left\{\psi_{k}\right\}$ of $H_{1, \mathfrak{h}^{\prime}, c}^{1}(D)$ consisting of eigenfunctions of $\mathscr{R}_{D, \mathfrak{h}^{\prime}}$. One can arrange that each $\psi_{k}$ has eigenvalue $\beta_{k}$ (by repeatedly listing the same $\beta_{j}$ as many times as its multiplicity, namely, $\operatorname{dim} E_{j}$ ). Thus

$$
\psi_{k}-\frac{1}{\beta_{k}} \mathscr{R}_{D, \mathfrak{h}^{\prime}} \psi_{k}=0 \quad \forall k=1,2 \cdots,
$$

so that $\psi_{k}$ is a solution to a Dirichlet Problem of the type (1.5) (with $g=0$ ), which is equivalent to solving the functional equation (6.1) (with $\lambda_{k}:=\frac{1}{\beta_{k}}>0$ and $\lambda_{k} \uparrow \infty$ ).

To prove the completeness of the system $\left\{\psi_{k}\right\}$ in $H_{1, \mathfrak{h}^{\prime}, c}^{1}(D)$, recall the fact that $\operatorname{ker}\left(\mathscr{R}_{D, \mathfrak{h}^{\prime}}\right)=\{0\}$. The desired conclusion follows then from the completeness criterion of [16, p. 234]. By a standard regularity criterion, if $\mathfrak{h}$ is of class $\mathscr{C}^{\infty}$ on $D^{*}$, then each eigenfunction $\psi_{k}$ belongs to $\mathscr{C}^{\infty}(Q)$ for all (open) domains $Q \Subset D^{*}$. Consequently $\psi_{k}$ belongs to $H_{1, \mathfrak{h},, c}^{1}(D) \cap \mathscr{C}^{\infty}\left(D^{*}\right)$.

Lemma 6.2. Given $g \in L^{2}(D)$, the Poisson problem (1.5) admits a solution $\psi$ in $H_{1, \mathfrak{\mathfrak { h } ^ { \prime }}, c}^{1}(D)$ if and only if the following functional equation on $\mathscr{C}^{\infty, c}(D)$,

$$
\left(I+\alpha \mathscr{R}_{D, \mathfrak{h}^{\prime}}\right) \psi=w
$$

with $w:=\mathscr{R}_{D, \mathfrak{h}^{\prime}} g \in H_{\substack{1, \mathfrak{h}^{\prime}, c \\ \text {, }}}^{1}(D)$, admits a solution $\psi$ in $H_{\substack{1, \mathfrak{h}_{\rightarrow}^{\prime}, c \\ 1}}^{1}(D)$.

Proof. The above equation (1.6) can be expressed alternatively as a functional equation on $\mathscr{C}^{\infty, c}(D)$ in the form

$$
[\psi, v]_{D, \mathfrak{h}^{\prime}}+\alpha\left[\mathscr{R}_{D, \mathfrak{h}^{\prime}} \psi, v\right]_{D, \mathfrak{h}^{\prime}}=[w, v]_{D, \mathfrak{h}^{\prime}},
$$

where $w:=\mathscr{R}_{D, \mathfrak{h}^{\prime}} g \in H_{1, \mathfrak{\mathfrak { h } ^ { \prime }}, c}^{1}(D)$. From this the equation (6.2) follows. 
Theorem 6.3. Assume that $(D, p)$ is a Rellich subdomain with respect to $\mathfrak{h}^{\prime}=(1, \cdots, 1)$. Let $g \in L^{2}(D)$. Consider the Poisson problem

$$
-\triangle_{p} \psi+\alpha \psi=g \text { a.e. in } D, \quad \psi \mid d D=0 .
$$

(A) If $\alpha \notin\left\{-\lambda_{j}\right\}_{j=1, \cdots, \infty}$ (the $\lambda_{j}$ being the eigenvalues of $-\triangle_{p}$ ), then there exists a unique weak solution $\psi \in H_{1,(0,1, \cdots, 1), c}^{1}(D)$ of the problem (6.3) with

$$
\|\psi\|_{1,(0,1, \cdots, 1)} \leq \text { Const. }\|g\|_{L^{2}(D)} .
$$

(B) If $\alpha=-\lambda_{j}$ (for some $\lambda_{j}$ as above), then the problem (6.3) has a weak solution $\psi \in$ $H_{1,(0,1, \cdots, 1), c}^{1}(D)$ if and only if $(g, \bar{\psi})_{D}=0$ for each $\psi=\psi_{j}^{k}, k=1, \cdots, s$, the latter being the associated eigenfunctions of the problem of Theorem 6.1:

$$
\left(I-\lambda_{j} \mathscr{R}_{D,(1, \cdots, 1)}\right)(\psi)=0 .
$$

Each solution of the the inhomogeneous problem (6.3) is of the form

$$
\psi=\psi_{0}+\sum_{k=1}^{s} c_{k} \psi_{j}^{k}
$$

where $\psi_{0}$ is a fixed solution and the $c_{k}$ are suitable constants.

Proof. In this proof let the allowable weight $\mathfrak{h}$ be $(0,(1, \cdots, 1))$. Since the Rellich embedding $\mathfrak{j}: H_{1,(0,1, \cdots, 1), c}^{1}(D) \hookrightarrow L^{2}(D)$ is compact, so is the composition $\mathscr{R}_{D,(0,1, \cdots, 1)} \circ \mathfrak{j}: H_{1,(0,1, \cdots, 1), c}^{1}(D) \rightarrow$ $H_{1,(0,1, \cdots, 1), c}^{1}(D)$. It is known that for a constant $\alpha \neq-\lambda_{j}$, the operator $I+\alpha \mathscr{R}_{D,(0,1, \cdots, 1)} \circ \mathrm{j}$ is invertible with a bounded inverse. Therefore the equation (6.2) has a unique solution

$$
\psi=\left(I+\alpha \mathscr{R}_{D,(0,1, \cdots, 1)}\right)^{-1} w_{1}
$$

with $w_{1}:=\mathscr{R}_{D,(0,1, \cdots, 1)} g \in H_{1, \underline{h}, c}^{1}(D)$, and

$$
\|\psi\|_{1, \underline{\underline{b}}} \leq\left\|\left(I+\alpha \mathscr{R}_{D,(0,1, \cdots, 1)}\right)^{-1}\right\|\left\|w_{1}\right\|_{1, \underline{\underline{h}}} .
$$

Since $\left\|w_{1}\right\|_{1, \underline{\underline{b}}} \leq$ Const. $\|g\|_{L^{2}(D)}$, the assertion in (A) is proved. To prove the assertion in (B), observe that the closure of the range of an operator is the orthogonal complement of the null space of its adjoint. The equation

$$
\psi-\lambda_{j} \mathscr{R}_{D,(0,1, \cdots, 1)} \psi=v_{1},
$$

where $v_{1}=\mathscr{R}_{D,(0,1, \cdots, 1)} g \in H_{1, \mathfrak{h}, c}^{1}(D)$, has a solution if and only if $v_{1} \in R\left(I-\lambda_{j} \mathscr{R}_{D,(0,1, \cdots, 1)}\right)$, which is equivalent to $v_{1} \perp \operatorname{ker}\left(I-\lambda_{j} \mathscr{R}_{D,(0,1, \cdots, 1)}^{*}\right)$. The latter means that $v$ is orthogonal (with respect to the inner product $[,]_{D,(0,1, \cdots, 1)}$ on $\left.H_{1, \mathfrak{l}, c}^{1}(D)\right)$ to all the eigenfunction $\psi_{j}^{k}$ corresponding to the eigenvalue $\lambda_{j}$, namely,

$$
\left[v_{1}, \psi_{j}^{k}\right]_{D,(0,1, \cdots, 1)}=\left(g, \bar{\psi}_{j}^{k}\right)_{D}=0
$$

The expression (6.4) is a consequence of the equation (6.5). 


\section{Acknowledgement}

The authors gratefully acknowledge the referee's suggestions, which have led to improvements of this paper. Initial inquiries concerning this work were started during a sabbatical granted by Dean B. Martensen of Minnesota State University, Mankato, to whom and the hosting institution, University of Padova, the first author expresses his best thanks. 


\section{References}

[1] A. Andreotti and W. Stoll, Analytic and algebraic dependence of meromorphic functions, Lecture Notes in Mathematics, vol. 234, Berlin-New York: Springer-Verlag, 1971.

[2] D. Barlet, Cycles analytiques complexes. I. Théorèmes de préparation des cycles, [Complex analytic cycles. I. Preparation theorems for cycles], Cours Spécialisés, vol. 22, Paris: Société Mathématique de France, 2014.

[3] H. Behnke and H. Grauert, "Analysis in non-compact complex spaces", in Proceedings of the Conference on Analytic functions (Edit. Behnke, H. and H. Grauert), pp. 11-44, Princeton Univ. Press, Princeton, N.J., 1960.

[4] R. Courant and D. Hilbert, Methods of Mathematical Physics, vol. I, New York: Interscience Publishers, 1962.

[5] J. Deny and J. L. Lions, "Les espaces du type de Beppo Levi", Ann. Inst. Fourier (Grenoble), vol. 5, no. 195, pp. 305-370, 1954.

[6] L. C. Evans, Partial Differential Equations, vol. 19, Providence RI: American Mathematical Society, 1998.

[7] F. Galaz-Fontes, "On Friedrichs inequality and Rellich's theorem", J. Math. Anal. Appl., vol. 145, no. 2, pp. 516-523, 1990.

[8] H. Grauert and R. Remmert, Theory of Stein Spaces, Grundlehren der Mathematischen Wissenschaften, vol. 236, Berlin-Heidelberg-New York: Springer-Verlag, 1979.

[9] H. Grauert and R. Remmert, Coherent Analytic Sheaves, Grundlehren der Mathematischen Wissenschaften, vol. 265, Springer, Berlin-Heidelberg-New York: Springer-Verlag, 1984.

[10] Ph. Griffiths and J. Harris, Principles of algebraic geometry, New York: John Wiley \& Sons, 1994.

[11] E. Hebey, Nonlinear Analysis on Manifolds: Sobolev Spaces and Inequalities, Courant Lecture Notes in Mathematics, vol. 5, New York University, Courant Institute of Mathematical Sciences, New York, Providence, RI: American Mathematical Society, 1999.

[12] M. Melgaard and G. Rozenblum, "Schrödinger operators with singular potentials", Stationary Partial Differential Equations (Edit. M. Chipot and P. Quittner), vol. II, Handb. Differ. Equ., Amsterdam, North-Holland: Elsevier, 2005, pp. 407-517.

[13] R. Narasimhan, Analysis on Real and Complex Manifold, Advanced Studies in Pure Mathematics, vol. 1, Paris: Masson \& Cie, Éditeurs, Amsterdam: North-Holland Publishing Co., New York: American Elsevier Publishing Co., 1968. 
[14] R. S. Palais, Seminar on the Atiyah-Singer Index Theorem, Annals of Mathematics Studies, No. 57, Princeton, New Jersey: Princeton University Press, 1967.

[15] F. Rellich, "Ein Satz über mittlere Konvergenz", Nachr. Ges. Wiss. Göttingen, Math.-Phys. Kl, pp. 30-35, 1930.

[16] F. Riesz and B. Sz.-Nagy, Functional Analysis, New York: Frederick Ungar Publishing Co., 1955.

[17] J. Ruppenthal, "Analysis on singular complex spaces", Habilitationsschrift, Fachgruppe Mathematik und Informatik, Bergische Universität Wuppertal, 2011.

[18] C. Tung, "The first main theorem of value distribution on complex spaces", Atti Accad. Naz. Lincei Mem., Cl. Sci. Fis. Mat. Natur. Sez. Ia, vol. 15, no. 4, pp. 91-263, 1979.

[19] C. Tung, "Semi-harmonicity, integral means and Euler type vector fields" 19, Adv. Appl. Clifford Algebr., vol. 17, no. 3, pp. 555-573, 2007.

[20] C. Tung, "On the weak solvability of Schrödinger type equations with boundary conditions", Math. Rep. (Bucur.), vol. 15 (65), no. 4, pp. 497-510, 2013.

[21] C. Tung, "On Wirtinger derivations, the adjoint of the operator $\bar{\partial}$, and applications". Izv. Ross. Akad. Nauk Ser. Mat., vol. 82, no. 6, pp. 172-199, 2018; translation in Izv. Math., vol. 82, no. 6, pp. 1239-1264, 2018.

[22] R. O. Wells, Differential Analysis on Complex Manifolds, Third edition, Graduate Texts in Mathematics, vol. 65, New York: Springer, 2008.

\footnotetext{
${ }^{19}$ For a corrected version of this paper, see: arXiv:1507.02675 [math.CV] DOI: 10.1007/s00006-007-0036-9.
} 